\title{
Lancet Commission on Global Surgery - A Public Health Initiative
}

\author{
Göran Kurlberg, MD, PhD
}

Department of Surgery, Sahlgrenska Academy, University of Gothenburg, Gothenburg, Sweden Research Department, Institute of Medicine, Tribhuvan University,Kathmandu, Nepal

\section{Corresponding author:}

\section{Göran Kurlberg, $M D, P h D$}

Department of Surgery, Sahlgrenska Academy, University of Gothenburg, Gothenburg, Sweden Research Department, Institute of Medicine, Tribhuvan University,Kathmandu, Nepal

Email: goran.kurlberg@surgery.gu.se

W ith the advent of the epidemiological transition, the incidence of noncommunicable diseases (NCDs) presenting as cardiovascular disease, diabetes, hypertension, malignancies and injuries make up the major part of the Global Burden of Disease..$^{1-3}$

Many of these conditions will require surgery and in 2015, the Lancet Commission on Global Surgery was launched. The Commission's key findings reveal that human and economic consequences of unmet surgical needs in low and middle-income countries (LMIC) are substantial and for many years have gone unrecognised. ${ }^{4,5}$

- Five billion people lack access to safe, affordable and timely surgical and anaesthesia care when needed.

- 143 million additional surgical and obstetric procedures are needed every year to save lives and avoid disability.

- Due to out-of pocket payments for surgery and anaesthesia 33 million people annually face catastrophic consequences for themselves and their family.

- On a national level, also for LMICs, investing in surgical services saves lives and promotes economic growth. Without necessary investments in surgical care LMICs will continue to have shortfalls in productivity estimated to USD 12.3 trillion between 2015 and 2030.

- Surgery, obstetrics and anaesthesia are indivisible, indispensable parts of all national health care systems.

There is, in many LMICs, a great disparity in access to safe, timely and affordable surgical and anaesthesia care between urban and rural areas. Ideally, surgical care starts in the community, where health workers connect patients in remote areas to different levels of providers. However, the possibility to receive surgical care depends on the accessibility and availability of surgical and anaesthetic providers who could deliver the care. ${ }^{6}$ There are different types of hindrances that could delay the patient from getting appropriate care. Accessibility to first level hospitals is often hampered by long distances and poor transportation. There may also be a lack of available resources both in equipment and trained personal in the nearby hospital. This will result in two different outcomes. The patients turn to informal providers (traditional healers), who are available and inexpensive. ${ }^{7,8}$ They may venture to travel to urban areas and attend tertiary level hospitals, resulting in overcrowded services in these settings. ${ }^{9}$

The Commission has developed a number of indicators to attack the deficiencies in health care systems regarding surgical and anaesthesia care. ${ }^{4,10-12}$

- $80 \%$ of the population in any area should be able to reach a hospital able to perform emergency procedures.

- All countries supplied with a workforce of at least 20 surgical, anaesthetic and obstetric physicians per 100000 population.

- $100 \%$ of the countries will be able to track surgical volume and perioperative mortality aiming at a $100 \%$ auditing.

- $100 \%$ protection against impoverishment from out-of-pocket payments for surgical and anaesthesia care. 
Achieving these indicators by 2030 will reach the aim of the Lancet Commission on Global Surgery of universal access to safe, timely and affordable surgical, anaesthesia and obstetric care when needed, a great public health initiative also in a country like Nepal.

\section{REFERENCES}

1. Arokiasamya P. India's escalating burden of noncommunicable diseases. The Lancet Global Health, 2018. 6(12): p. e1262-e1263.

2. Bloom DE, Cafiero ET, Jané-Llopis E et al. The global economic burden of noncommunicable diseases. World Economic Forum, Geneva. 2011

3. Wahdan $\mathrm{MH}$. The epidemiological transition. Eastern Mediterranean Health Journal„»1996. 2(1): p. 8-20.

4. Meara JG, Leather AJM, Hagander $L$ et al. Global Surgery 2030: evidence and solutions for achieving health, welfare, and economic development. Lancet, 2015. 386(9993): p. 569624.

5. Bickler S, Ozgediz D, Gosselin R et al. Key concepts for estimating the burden of surgical conditions and the unmet need for surgical care. . World J Surg 2010. 34(374-80).
6. Grimes $\mathrm{CE}$, Bowman KG, Dodgion $\mathrm{CM}$ et al. Systematic review of barriers to surgical care in low-income and middle-income countries. World J Surg 2011. 35: p. 941-50.

7. Bergström S, Goodburn E. The role of traditional birth attendants in the reduction of maternal mortality In: Safe motherhood strategies: a review of the evidence., V.L.W. De Brouwere V, Editor. 2001, ITGPress: Antwerp, Belgium. p. 77-95.

8. Bloom G, Standing $\mathrm{H}$, Lucas $\mathrm{H}$ et al. Making health markets work better for poor people: the case of informal providers. Health Policy and Planning, 2011. 26(1): p. i45-i52.

9. Rehmani R. Emergency section and overcrowding in a university hospital of Karachi. J Pak Med Assoc, 2004. 54: p. 233-37.

10. Hammer J, Jack W. Designing incentives for rural health care providers in developing countries. J Dev Econ, 2002. 69: p. 297-303.

11. Holmer $H$, Lantz $A$, Kunjumen $T$ et al. Global distribution of surgeons, anaesthesiologists, and obstetricians. Lancet Glob Health., 2015. 3: p. S911.

12. Lagomarsino G, Garabrant A, Adyas A et al. Moving towards universal health coverage: health insurance reforms in nine developing countries in Africa and Asia. Lancet 2012. 380: p. 933-43. 